\title{
Bangabandhu satellite-1: A remarkable feat of a nation
}

\section{Md Mizanuzzaman}

\author{
Hussain Nyeem \\ Military Institute of Science and Technology (MIST) \\ Mirpur Cantonment, Dhaka-1216, Bangladesh \\ mizan zaman@yahoo.com \\ h.nyeem@eece.mist.ac.bd
}

\begin{abstract}
Bangabandhu Satellite of Bangladesh is the pioneering and the most remarkable achievement of the country. This dream of a nation, however, has not come easy. An incredible long-term planning and initiatives now put the footprint of the country-Bangladesh on the space. This article reports that incredible contributions of a nation, pragmatic vision and initiatives of the father of the nation, and the promises and future opportunities of this achievement. It also captures a few important historical notes on space satellite communication and the then socioeconomic condition of the country. The future scope and better utilization of the satellite in all possible aspects have been primarily considered and presented in support of the development of a more effective roadmap of the country's overall communication sector.
\end{abstract}

\section{Introduction}

The country's first communication satellite, Bangabandhu Satellite-1 (BS-1), has already started revolving around its orbital slot in space. With this satellite, Bangladesh has become the 57th country in the world and fourth in South Asia, after India, Pakistan, and Sri Lanka, to have its nationally-owned satellite in space. The satellite was manufactured by Thales Alenia Space, launched on 12 May 2018 at $119.1^{\circ}$ E longitude, and named after The Father of the Nation, Bangabandhu Sheikh Mujibur Rahman.

The visionary leader, Bangabandhu Sheikh Mujibur Rahman, established the earth station in Betbunia to develop the information technology sectors in line with the developed countries in the year 1975. With that initiation and in the process of 'progress, Bangladesh has obtained the communication satellite, BS-1. This communication satellite is a crucial milestone in the country's scientific paradigm and a big step towards achieving self-dependency.

The BS-1 would deliver a much-needed boost to the country's digital advancement, economic growth, job creation, and a wide range of services indifferent sectors like communication, disaster, socio-economic, and defense sectors. Besides providing Internet to rural communities, it would bring enormous potential and possibilities to 
improve agriculture, execute disaster planning, and ensure national security. It has also created an environment for interest in space science and satellite technology.

\section{The Beginning of Communications Satellites}

Satellite communication is no longer a new idea. It was introduced by the American writer Edward Everett Hale in his story "The Brick Moon" in 1869. Later, Arthur C. Clarke developed a more realistic concept in 1945. Clarke introduced the geostationary orbit at an altitude of 35,786 km (22,236 miles) above the Earth's surface. Communication with the geostationary satellite is considered ideal because it has no continuous tracking of the satellite's position from a base station. The first artificial satellite, Sputnik 1, was launched by the Soviet Union on 4 October 1957. US National Aeronautics and Space Administration (NASA) with AT\&T's Bell Labs stepped in with their initial Echo 1 and Echo 2 satellites launched on 12 August 1960 and 25 January 1964, respectively. Built upon the ground signal reflection principle, the Echo 1 and Echo 2 satellites were discontinued as the passive satellites.

As a full-fledged active satellite, Telstar 1 was successfully launched on 10 July 1962 to offer two-way communications like live-telecast and phone-call. Unlike the Telstar 1 placed into the low Earth orbit, the pioneering Syncom 1 was launched in the geostationary orbit on 14 February 1963, which was missing soon after its launch. Thus, the next generations of Syncom were successfully launched in successive years. Syncom 2 was the first satellite in a geosynchronous orbit (an orbit inclined to the Equator having a period of 24 hours), and Syncom 3 was the first geostationary satellite. Canada was the third country that launched its communications satellite, Anik 1, on 9 November 1972. Indonesia follows the parallel development with its Palapa 1 satellite launched on 8 July 1976. After that, the pursuit of developing and launching satellites has been popular worldwide. These earlier developments of communication satellite technology paved the way for a global communications satellite industry.

\section{Rise of a Nation with War-ravaged Economy}

While many developed countries began to realize space communications and technology in the middle of the nineteenth century, our nation struggled to proclaim our sustainable cultural and geographical identity. In a few successive decades from 1957, countries like the Soviet Union, US, and Canada developed and launched their satellites. On the other hand, Bangladesh achieved its identity on 16 December 1971. Besides, while hundreds of billion dollars' space research programs were running by those developed countries, Bangladesh inherited an economy of only eight billion US dollars after the liberation war [1]. However, with the committed leadership of Bangabandhu Sheikh Mujibur Rahman, the dire economic situation started reviving against overwhelming defies, including war- ravaged socio-economic structures and lacking any progressive regulatory institutions. 
Bangabandhu, the visionary leader, had correctly identified the primary problems and the potentials of his nation to turn the tide of the economy. The people are led to rebuilding the shattered economy with highly limited domestic resources and international humanitarian support. Achieving agricultural sustainability and adequate industrialization are correctly prioritized to drive socio- economic development. Agriculture was considered to feed the people and enable sustainable industrialization.

Sustainable industrialization without indigenous entrepreneurial experience and technical knowledge was highly ambitious for the nation when there was an extreme scarcity of foreign reserves and investments. Thus, Bangabandhu attempted to fill that gap with a new and pro-science education policy and prioritized education in the first Bangladesh Constitution. Planning for developing an enlightened nation was reflected in different free compulsory primary education initiatives and new universities' establishment, including medical and technical universities.

Nevertheless, while concentrating on building a nation from scratch, Bangabandhu did not overlook the world's concurrent technological progress. In addition to building a skilled workforce, his few other initiatives also demonstrated his futuristic strategy to befit the nation with the advances in science and technology. To immediately derive maximum benefits of the third industrial revolution, membership of the International Telecommunication Union (ITU) was immediately ensured. The country's first satellite ground station was established at Betbunia under Kawkhali Upazila of the Rangamati district to get the nation connected worldwide with data- and telecommunications. The earth station was inaugurated by Father of the Nation Bangabandhu Sheikh Mujibur Rahman on 14 June of 1975. The station is situated on 128 acres at Betbunia beside the Rangamati-Chattogram road to connect the world with Bangladesh through a satellite located over 35,900 kilometers high from the earth station.

\section{The Golden Renaissance and a National Pursuit to Touching the Space}

Bangladesh has been undergoing a radical transformation age, which may be called 'the golden renaissance'. Following the father of the nation's envisaged tracks for sustainable development, his able daughter, The Honorable Prime Minister Sheikh Hasina, propels the country towards prosperity. In a decade (from 2009 to present), the country's digital transformation has successfully been initiated. It is brought to a new height with the state-owned geostationary communication satellite, BS-1. As the 57th country in the world, the BS-1 is adorned with the Bangladesh flag and 'Joy Bangla' insignia on it, endorsing the nation on the space.

Like all the developed countries having their satellite in orbit, Bangladesh launched its satellite to reduce its dependency on the other countries to pursue sustainable development. Bangladesh Telecommunication Regulatory Commission (BTRC) started working with the consultation of USA based consultancy firm Space Partnership 
International (SPI) and specialist advice of the International Telecommunication Union (ITU) for shifting the telecommunication sector of Bangladesh to a new paradigm by launching its first-ever satellite. The satellite would have a crucial role in enabling sustainable and uninterrupted telecommunication services in Bangladesh over the greater risk of a natural disaster.

Realizing a state-owned satellite's long-cherished dream may be regarded as the most remarkable national pursuit of a complete digital transformation. Following the ITU regulations, the BTRC committee started with lodging the Coordination Request (CR) for the $1020 \mathrm{E}$ and $690 \mathrm{E}$ to ITU and all successive liaisons with ITU [10]. This project was supported by a USA based consultancy firm, Space Partnership International (SPI). The project team and SPI carried out the feasibility study, market analysis, business plan, determination and development of an overall communications system, and desirable performance characteristics for the satellite. Besides, attaining other requirements like the coordination agreements, recommended orbital slot and frequencies, design and development of the satellite system architecture, and orbit test were also performed.

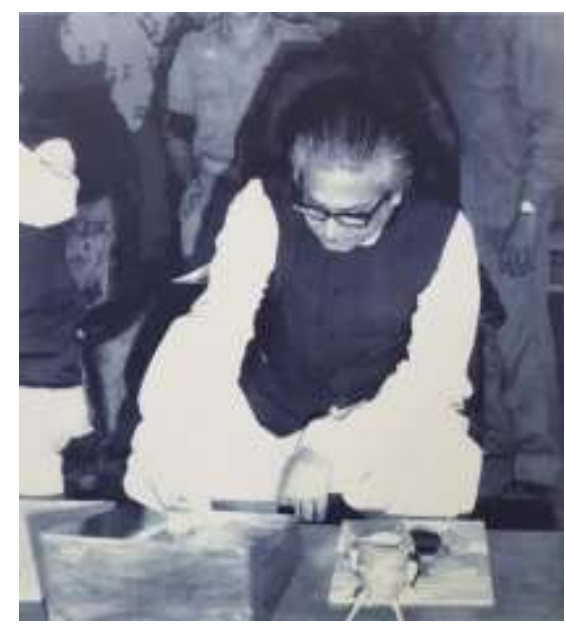

Figure 1. Bangabandhu officially opeining the Betbunia Satellite Station by pressing the start button.

Source:https://bangabandhumuseum. org.bd/en/gallery/details/54/21

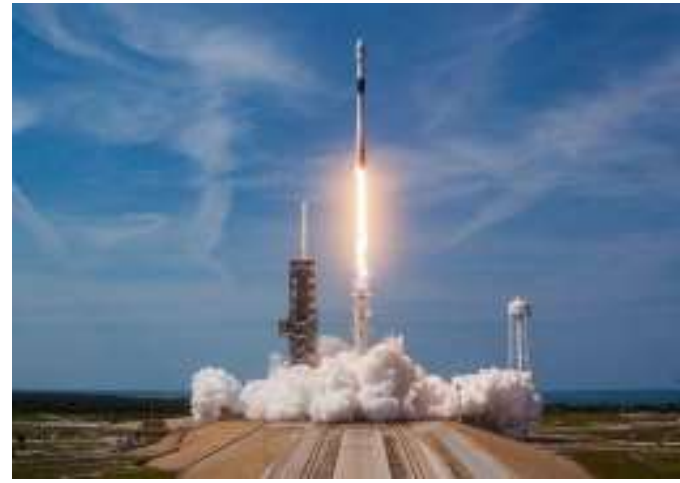

Figure 2. Falcon 9 carrying BS-1 satellite during launch.

Source:https://www.flickr.com/photos/space $x / 42025498972 /$ 


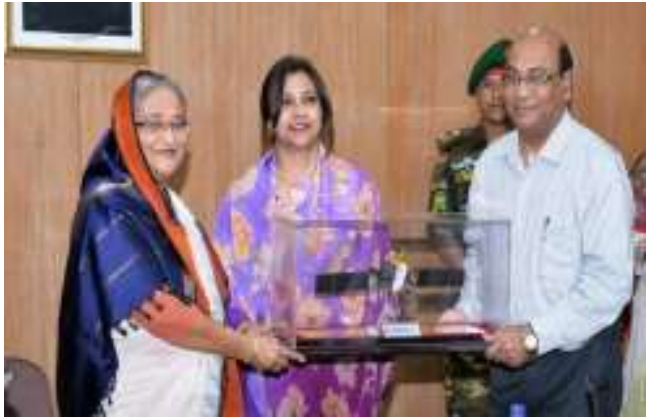

Figure 3. Prime Minister Sheikh Hasina is receiving a replica of BS-1, April 17, 2017.

Source:https://www.thedailystar.net/ country/pm-receives-replica-bangabandhu satellite-1392238

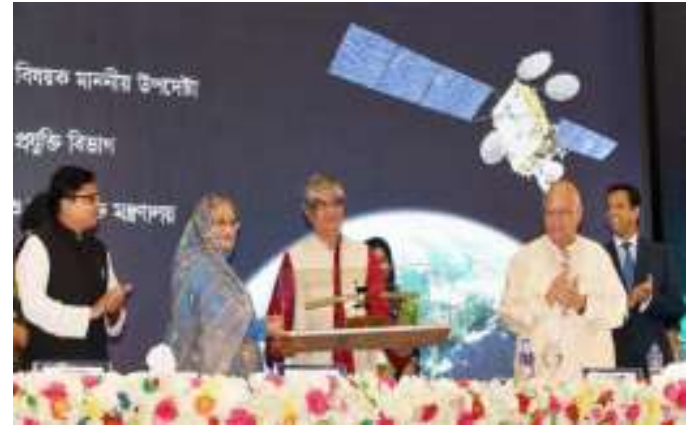

Figure 4. Prime Minister inaugurated the commercial transmission of all local private television channels using the feed of BS-1.

Source:https://www.bangladeshinfo.com/ article/2054/

According to the BTRC project plan, the BS-1 was finally constructed with the Spacebus4000B2 and launched in May 2018 as the first geostationary communications satellite of Bangladesh [2]. Its first payload was launched by a Falcon 9 Block 5 rocket of SpaceX from Kennedy Space Center on Merritt Island, Florida, USA [3]. A government-owned agency, Bangladesh Communication Satellite Company Limited (BCSCL), was formed for the operation and control of BS-1 [4]. The ground control stations: primary station in Gazipur and secondary station at Betbunia, Rangamati, were established by Thales Alenia Space with its partner Spectra.

\section{Prevailing Services of Bangabandhu Satellite-1 (BS-1)}

The BS-1, a communication and broadcasting satellite, is designed to be operated with $24 \mathrm{Ku}$ and $16 \mathrm{C}$-band transponders. The satellite enables the nation to save approximately BDT 1.08 billion (USD 14 million) spent on satellite rents a year [5]. BTRC also plans to launch follow-on series of BD-2 and BD-3 satellites in phases. However, as illustrated in Figure 5, the BTRC prioritized satellite applications are Direct to Home (DTH), VSAT, Backhaul and Trunking, Network Restoration, Disaster Preparedness, and relief [6]. The desired potential services of BS-1 are briefly discussed below. 


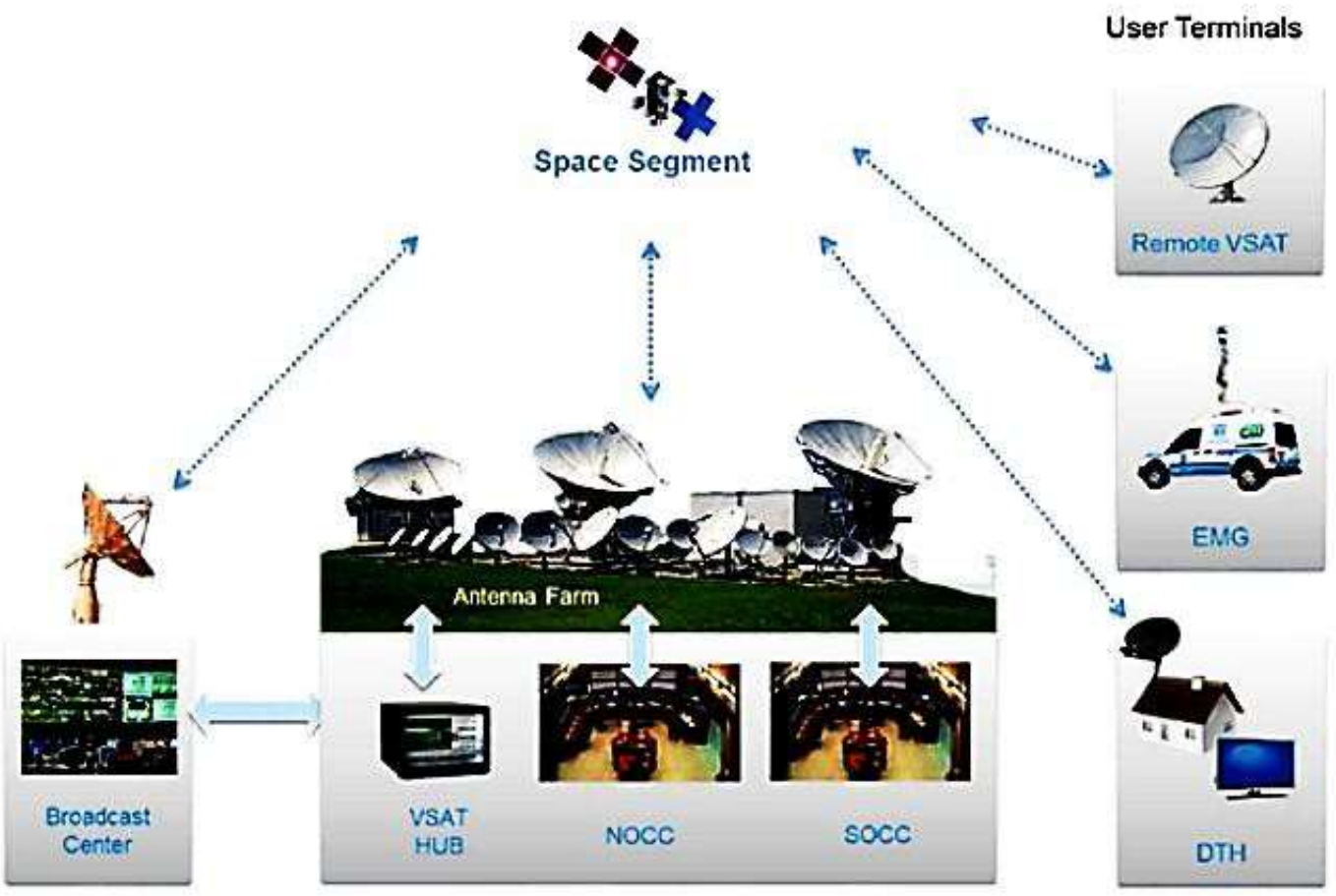

Figure 5. Conceptual model of BS-1 Source: https://www.btrc.gov.bd/

1. DTH. The BS-1 would provide the Ku-band home service destined to small-sized antennas capturing radio and data service and multiplexed digital television service. Usually, the operators in the respective countries offer these services. The services' content is packaged at their broadcast center(s) to transmit them to the Bangabandhu Ground Station or another earth station facility to uplink the satellite [3].

2. Video Distribution. This service utilizes the C-band transmission to medium-sized antennas anywhere in the satellite's footprint. This service mainly includes video transmission for multiplexed digital television and data services. These service users are likely to be broadcasters distributing their content services to agents like cable TV network operators or re-broadcasters like DTH Operators in other countries [3].

3. VSAT Private Networks. The BS-1 would establish private networks to offer multimedia services like voice, data, video, and Internet-based communications to banks, corporate offices with medium-sized antennas. These services will be delivered to Bangladesh using Ku-Band and the regions captured by India Plus using C-Band beams. 
4. Broadband. In addition to the VSAT private networks, dedicated Ku- Band Broadband service would also be offered to the end-user (individual, organization, corporation, or government) to get remote access to the Internet at high speed and high quality of service.

5. Communications Trunks. For the development of dedicated digital infrastructure, the Ku-Band and C-Band based high data-rate communication trunks would enable the point-to-point services with large antennas. Typically, telecommunication companies and Global System for Mobile Communications (GSM) operators use these services to promote e-learning, telemedicine, and distant office-works services. An uninterrupted telecommunication service in national or regional natural calamities can also be established.

\section{National and Defense Prospect with BS-1}

In addition to the desired commercial services, the BS-1 would also help promote different national and defense sectors, including the broadcasters, Internet service providers (ISPs), governments, and the military. To this end, the communication services of BS-1 can be classified into three main groups: telecommunications, broadcasting and data communications. These primary and special services are briefly discussed below in light of the national and defense prospect.

1. Communications Services. Communication services are as follows:

a. Telecommunications. The primary telecommunication services include telephone calls and GSM services provided to telephone companies and wireless and cellular network providers. Further to these services, satellite communications technology could also provide adequate support to tackle natural disasters and emergencies with mobile satellite equipment deployed to disaster areas.

b. Broadcasting. This service captures the radio, television, and mobile broadcasting services delivered directly to the consumer and local stations, largely affiliating the satellite. Satellites usually distribute signals from one point to multiple points. Thus, it is ideal for "point-to-multipoint" communications like broadcasting. Unlike the other ground-based communication technology, no massive ground infrastructure is required for satellite communication leaving a greater prospect to this country having underserved and isolatedareas.

c. Data-communications. This service involves point-to-point data transfer. This service type is greatly facilitated by using very small- aperture terminal (VSAT) 
networks with the satellite. It is used by corporations and organizations that require financial and other information to be exchanged between their various locations. With the Internet's growth, a significant amount of Internet traffic goes through satellites, making ISPs one of the largest customers for satellite services.

2. Special Services. Better utilization of the communication services discussed above would enable different BS-1 services to support the national and social developments.

a. Remote Sensing. The BS-1 would support collecting data from the Earth's surface and water bodies. Thereby, the collected data may be studied for better agricultural land management, water management, flood-affected land estimation, and earth-surface-related investigations.

b. Disaster Management. The continuous collection and analysis of meteorological information from BS-1 would facilitate more effective forecasting of any disaster. The satellite would also be an effective medium of any warning dissemination concerning disaster management centers. Again, during unexpected disasters situation, the general Telecommunication system may be disrupted. In that situation, the Satellite network can play an essential role in the country's uninterrupted Telecommunication service.

c. Distant Training and Development. A more connected hub can be created for distant training and development in all districts and union territories. Such a satellite-based multimedia communications hub would offer more effective teaching and interactive remote classrooms in isolated and remote areas and promote universal education. Again, online academic classes and training also can be conducted more effectively all over the country during COVID-19 or future pandemic situations.

d. Tele-medicine. This service would enable the rural healthcare sector more reachable and connect the hospitals and community health centers to the disadvantaged and underserved populations in the rural areas. It is the desired application of space technology for societal development with expert consultation through medical software integrated with computer hardware and medical diagnostic instruments. During future pandemic like the COVID19 situation, it can be utilized when general communication service is unavailable or less effective.

e. Community Resource Center. Satellite-based communication technologies can be brought further to provide services related to education, 
healthcare, natural resources management, and the impact of natural disasters. The BS-1 may support programs to set up Community Resource Centers with a single-window delivery mechanism for various space-based products and services directly to the rural areas. These services primarily include telemedicine, natural resource management, and interactive advisories on agriculture, fisheries, land and water resources management, livestock management. Besides, interactive vocational training towards alternative livelihood, e-governance, and weather information may also be supported by the BS-1 services [7].

f. Space Research and Exploration. Nevertheless, the BS-1 would create a new paradigm for Bangladesh with new space research exploration opportunities. Bangladesh Space Research and Remote Sensing Organization (SPARRSO) are recently formed to extend the services and development of next-generation BS to take pride in space technology.

3. Military Services. Besides the special services for national development, different services of BS-1 may also be developed for the Bangladesh Armed Forces division. A few of such special services are noted below.

a. Dedicated Communications. Military operations may not be thought of without a reliable communication system. The BS- 1 would support establishing reliable communication for military use indigenously. Usually, military operations are carried out in vast areas with some poor telecommunications infrastructure. The telecommunication service and reserved communication trunk with modern satellite communications systems may be used with the military-grade communication systems. This arrangement would provide fast, reliable, interference- and interceptionresistant information transfer in command centers, operating units, and other sub-units. The Signals Corps of Bangladesh Army may take this opportunity for widening its service for itself and other Army units deployed in different areas with the poor conventional communication network system.

b. Remote Command and Control. Existing military operations, peacekeeping, and stabilization missions need the armed forces to take actions in unknown and outlying regions. Because of Armed Forces in mission operations on other continents having poor telecommunications infrastructure, satellite communications remain the only possible option.

c. Remote Access with Space Technology. With emerging technologies like the Internet of Things (IoT), cloud computing, and artificial intelligence, electronic 
control with remote access can be of paramount importance these days for Bangladesh. Consequently, the BS-1 would facilitate this service with datacollection and transmission of control messages from sensors to actuators for military operations. Thus, the loT sensors and actuators are distributed over different formations or units, many of which are located in remote areas and are not served by terrestrial access networks. Thus, the BS-1 can offer interoperability between satellite systems and sensors/actuators and internet connectivity over satellite- based and group-based communications. Besides, the development of radio resource management systems is a prerequisite to improving loT over satellite.

d. Space Positioning and Mapping Systems. Accurate location of the enemy and own forces is a critical requirement for military command and operations. Timely dissemination of that information can thus highly facilitate the recent electronic battlefield. This nation's new space-age has led to the deployment of state-of-the- art technologies, including Global Positioning System (GPS) [8] and Deep-Space Positioning System (DPS) [9]. The military forces use GPS technology for diverse applications during wartime and peacetime, including navigation, targeting, rescue, guidance, and facility management [10]. A further development of GPS for Deep Space called DPS could heighten the armed forces' technological strength and capability with deep-space navigation and target-relative navigation. These DPS applications can be implemented by placing the wide- and narrow-angle cameras, and satellite navigation imaging, and radiometric link closure without the aid of artificially provided infrastructure [10]. Field Arty and AD Regiment of Bangladesh Army easily collects the own and enemy's global positioning data/ digital grid reference and the Meteorological data required for destruction of enemy or enemy's aircraft. MLRS/ SP Guns have the opportunity to use this data for more effectiveness. Bangladesh Air Force and Navy also can utilize these data from BS-1 to destroy the enemy in the sky or sea.

e. Guidance System. The guidance system would be taken to a new height with BS-1 services and modern communication technology. A guidance system may also be considered a part of a navigation and control system. It is more dedicated to independently control the movement of a ship, aircraft, missile, rocket, satellite, or any other moving object by determining the changes in position, velocity, altitude, and rotation rates of a moving object through a specific trajectory and altitude profile [11]. This system highly relies on sensors like compasses, GPS receivers, star trackers, inertial measurement units, and altimeters. The environmental conditions and vehicle characteristics may also affect the system's accuracy. Thus, the BS-1 could be an essential component to transmit the dynamic information about the surrounding 
environment to the control system of a guidance system for self-adjustment of the object's flight path and orientation.

\section{Conclusion}

The father of the nation's contribution to the planning and establishing a stable socioeconomic backbone of Bangladesh remains an undeniable and the most significant impulse for the ongoing national pursuit of digital transformation. Among many commendable national initiatives by the present government, the able daughter of Bangabandhu, the BS-1, is possibly the most remarkable one after liberation, which holds the nation's footprints on the space and justifies this age for the 'Golden Renaissance'.

The BS-1 has begun a new era of the country's digital transformation with many new services and opportunities to take the socio-economic state to a newer height. Besides saving and earning foreign currencies, the BS-1 would improve the capacities of the television channels, mobile phone companies, DTH TV service providers, internet connectivity, and weather forecast. The communication services would enable new scopes of many applications with cutting-edge technologies like loT, cloud computing, artificial intelligence, and deep-space positioning. Thus, both the national and military sectors would benefit from those applications, including telemedicine, cooperative learning, distant training, disaster management, community resource centers, and autonomous navigation and guidance system.

Despite the enormous promises and possibilities of BS-1, better utilization and management services also require the extensive development of the groundcommunication infrastructure, deployment of cutting-edge technologies, development of low-cost reusable launch vehicles, and initiatives to ensure wider bandwidth on demand. Such developments in forward-marching Bangladesh also promote the Bangabandhu satellite series as a profitable asset with long-term commercial viability. With the legacy of Bangabandhu, innovative initiatives of his able daughter, and the promises and possibilities of BS-1, seeking a developed economy for Bangladesh by 2041 can no longer be highly ambitious at the age of Mujib Barsho.

\section{References}

1. https://thefinancialexpress.com.bd/special-issues/special-on-national-mourningday/bangabandhus-thoughts-on-development-focus-on-industrialisation1534260014.

2. https://www.aerospace-technology.com/projects/bangabandhu-1-bd-1communication- satellite/. Consulted on 18 October 2020.

3. "SpaceX launches Bangabandhu-1 with most powerful Falcon 9 rocket". The Daily Star,13 May 2018. Retrieved on 19 May 2018. 
4. Official Website of Bangabandhu Satellite -1 Operator Company: Bangladesh Communication Satellite Company Limited (BCSC), 3 March 2018.

5. http://www.btrc.gov.bd/rti-designated-officer-0. Consulted on 25 October 2020.

6. http://bcscl.com.bd/pages/9.

7. Nair, G.M., (2008), "Role of communications satellites in national development", IETE Technical Review, Volume: 25, Number: 1, pp. 3-8.

8. http:/www.loc.gov/rr/scitech/mysteries/global.html.

9. NASA Technology Transfer Program, "Deep-Space Positioning System (DPS)", from https://technology.nasa.gov/patent/NPO-TOPS-26.

10. McDuffie and Juquai, "Why the Military Released GPS to the Public", Popular Mechanics, 19 June 2017.

11. Draper, Wrigley, Hoag, Battin, Miller, Koso, Hopkins and Vander Velde, "Apollo Guidance and Navigation (Report)", Massachusetts Institute of Technology, Instrumentation Laboratory, June 1965, Retrieved on 12 October 2014. 